\title{
Research on Non-stationary Blind Separation Method for Unfixed Signal Based on Extended Joint Diagonalization
}

\author{
LI Jun ${ }^{1}$,Chen Xin-Yuan ${ }^{2}$ \\ \{lijun73812@163.com¹, chenxinyuan7559@163.com²\} \\ (1.College of Artificial Intelligence,Jiangxi Institute of Applied Science and \\ Technology,Nanchang 330100, China; \\ 2.College of Artificial Intelligence,Jiangxi Institute of Applied Science and \\ Technology,Nanchang 330100,China)
}

\begin{abstract}
In order to solve the problem of signal instability caused by the number of iterations of the traditional nonstationary signal underfixed blind separation method, a nonstationary signal underfixed blind separation method based on extended combined diagonalization method is studied. Firstly, the second-order correlation matrix is extended to the fourth-order accumulation by the extended joint diagonalization method, the linear time-frequency variation of the nonstationary signal is analyzed, the uncertain part of the nonstationary signal is calculated, the function expression of the source signal is obtained, and the time-domain and frequency-domain changes in the function expression of the iterative source signal are obtained. The experimental results show that compared with the traditional separation method, the nonstationary signal underfixed blind separation method based on extended joint diagonalization method has the least number of iterations, and the isolated signal is the most stable.
\end{abstract}

Keywords: Extended joint diagonalization; nonstationary signal; underfixed blind separation; number of iterations

\section{Introduction}

With the development of information technology, radar, communication and other electronic equipment are increasingly widely used, and the frequency band of use is also expanding. In addition, the complex and changeable topography, the movement of signal source caused by the transfer of personnel and equipment, and the unintentional interference caused by artificial intentional interference and various natural radiation, these factors form a complex electromagnetic environment with 
high density in time domain, severe aliasing of spectrum, interlaced space and dynamic variation of time ${ }^{[1]}$. In the complex electromagnetic environment, there are other unknown interference signals in the mixed signals received by radar, communication and other cooperative systems, besides their own signals, which seriously affect the normal operation of the system ${ }^{[2]}$. For non-cooperative systems, because the receiver receives wide beam and wide bandwidth coverage, the intercepted multiple signals are often overlapped in time domain, frequency domain and even airspace, so it is difficult to complete the reconnaissance processing tasks such as parameter estimation and information extraction directly at this time. Moreover, because the number of potential source signals is unknown and the number of array elements is limited, the number of sources in the mixed signal is often larger than the number of array elements ${ }^{[3]}$. Therefore, either the cooperative system or the non-cooperative system usually need to be separated by docking the received hybrid signal first.

In the complex electromagnetic environment, because the mixed signals are overlapped in time domain and frequency domain, the separation method based on traditional time domain or frequency domain filter cannot adapt to this complex situation ${ }^{[4]}$. With the development of array signal processing technology, spatial filtering based on signal wave direction estimation and beamforming has become a major means of multi-signal separation ${ }^{[5]}$. However, the method of spatial filtering has specific arrangement requirements for antenna arrays and must have known accurate array parameters. However, it is difficult to obtain the exact parameters about the array in a non-cooperative environment, which limits the practical application of the algorithm. When the number of sources in the hybrid signal is larger than the number of array elements, even if the array parameters are known, the method of spatial filtering cannot complete the separation of the signals .

At present, blind signal separation is the main method to solve this problem. It can recover the waveform of the source signal from the observed hybrid signal under the condition of unknown mixing process and source signal. Separation technology, which originated from the study of cocktail parties. Cocktail party sound separation technology refers to in the noisy environment, the voice source signals issued by different speakers, mixed together through space transmission, the human ear can accurately capture the voice of concern and interest. The separation technology only needs the source signal to satisfy the few assumption conditions (e.g. sparsity, independence, non-Gaussianity, etc.), the source signal can be recovered by using the 
observation data of each array element, which has strong adaptability.

\section{Research on Non-stationary Blind Separation Method for Unfixed Signal Based on Extended Joint Diagonalization}

\subsection{Using Extended Joint Diagonalization Method to Calculate Signal Tensor}

When the signal tensor is established by the extended joint diagonalization method, the nonstationary signal is in the transient mixed form, and the mixed matrix is nonnegative. A model of the signal separation of the underfixed blind source is established, as follows:

$$
\left[\begin{array}{c}
x_{1}(t) \\
x_{2}(t) \\
\vdots \\
x_{m}(t)
\end{array}\right]=\left[\begin{array}{cccc}
a_{11} & a_{12} & \cdots & a_{1 n} \\
a_{21} & a_{22} & \cdots & a_{2 n} \\
\vdots & \vdots & \ddots & \vdots \\
a_{n 1} & a_{n 2} & \cdots & a_{n n}
\end{array}\right]\left[\begin{array}{c}
s_{1}(t) \\
s_{2}(t) \\
\vdots \\
s_{n}(t)
\end{array}\right]+\left[\begin{array}{c}
e_{1}(t) \\
e_{2}(t) \\
\vdots \\
e_{m}(t)
\end{array}\right]
$$

In the formula, $X(t)=\left[x_{1}(t), \ldots, x_{m}(t)\right]^{T}$ is the observation signal vector, its signal number is $m, S(t)=\left[S_{1}(t), \ldots, S_{n}(t)\right]^{T}$ is an unknown source signal vector, the number of signals is $n$, and $m<n \cdot A=\left[a_{1}, \ldots, a_{n}\right]$ is the unknown instantaneous mixing matrix of $m \times n \cdot E(t)=\left[e_{1}(t), \ldots, e_{m}(t)\right]^{T}$ denotes Gaussian white noise. The white noise has little effect on the source signal when the higher order accumulator of the signal is obtained, so the formula (1) can be reduced to:

$$
X(t)=A S(t)
$$

The second-order correlation matrix of the nonstationary signal obtained by constant transformation upper formula (2) is as follows:

$$
\begin{aligned}
R_{x x} & =E\left[X(t) X(t)^{T}\right]=E\left[A S(t) S(t)^{T} A^{T}\right] \\
& =A E\left[S(t) S(t)^{T}\right] A^{T}
\end{aligned}
$$

In the formula, $E$ means mathematical expectation. For the second-order correlation matrix of the upper (3) nonstationary signal, the implied semaphore is less than the fourth-order accumulation, and the signal estimation is easily disturbed by Gaussian noise. The interference signal is shown below: 


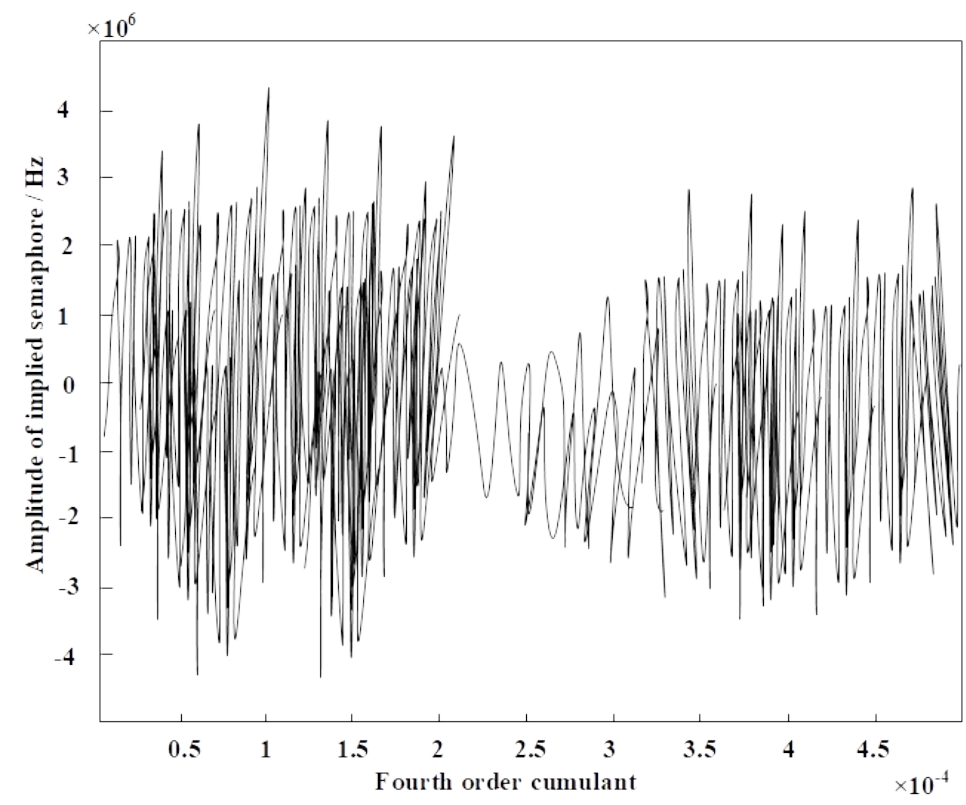

Fig.1 Non-stationary signal disturbed

To avoid the problem in the figure above, the second-order correlation matrix is extended to fourthordercumulant.

$$
Q^{X}=\operatorname{cum}\left\{X, X^{*}, X^{*}, X\right\} \in R^{m^{2} \times m^{2}}
$$

In the formula, $Q^{X}$ indicates the fourth order of the signal. The signal tensor is a nonnegative value when the mixed matrix is nonnegative in a certain physical sense according to the fourthordercumulant property. Let the nonnegative tensor be:

$$
Y=B C^{T}
$$

In the formula, $B=\left[b_{1}, b_{2}, \ldots, b_{j}\right]$ and $C=\left[c_{1}, c_{2}, \ldots, c_{j}\right]$ are non-negative matrices. Calculate the Euclidean distance of formula (5):

$$
J\left(b_{1}, b_{2}, \ldots, b_{j}, c_{1}, c_{2}, \ldots, c_{j}\right)=\frac{1}{2}\left\|Y-B C^{T}\right\|^{2}
$$

The residuals in the non-negative matrix are defined and calculated to:

$$
Y^{(j)}=Y-\sum_{p \neq j} b_{p} c_{p}^{T}
$$


The formula for calculating the tensor of a nonstationary signal is as follows:

$$
Q^{r}=\frac{R^{m^{2} \times m^{2}} B C^{T}}{Y-\sum_{p \neq j} b_{p} c_{p}^{T}}
$$

The tensor of the nonstationary signal calculated from formula (8), the linear time-frequency variation of the nonstationary signal is analyzed, and the blind separation method of the nonstationary signal is studied ${ }^{[6]}$.

\subsection{Analysis of linear time-frequency variation of nonstationary signals}

When analyzing the linear time-frequency variation of nonstationary signal, the linear superposition of Frequency hopping source signal $S_{1}(t)$ and $S_{2}(t)$ is adopted.

Using the time-frequency analysis method to process the hopping source signal, the time-frequency representation of the hopping source signal can be obtained as follows:

$$
T F R_{s}(t, f) 、 T F R_{s_{1}}(t, f) 、 T F R_{s_{2}}(t, f) \text {, The processed frequency }
$$
hopping signal still conforms to the linear superposition principle. The formula is as follows:

$$
\operatorname{TFR}_{s}(t, f)=\operatorname{aTFR}_{s_{1}}(t, f)+b T F R_{s_{2}}(t, f)
$$

On the basis of the above formula, the window function is translated on the time axis, and the source signal is divided into several short-time signals, Then Fourier transform each short-time signal so that the frequency of communication signal can be obtained in different time periods. Take Observation signals $x(t)$ as an example,

The continuous STFT and its inversion are expressed as:

$$
\operatorname{STFT}_{x}(t, f)=\int_{-\infty}^{+\infty} x(\tau) h^{*}(\tau-t) e^{-j 2 \pi f \tau} d \tau
$$

In the formula, $h^{*}(\cdot)$ means window function. By multiplying the observation signal with the window function, the Fourier transform is carried out. The timefrequency resolution of short-time Fourier transform (STFT) is therefore related to the nature of the window function, the width of the window function is inversely proportional to the time resolution. And the selection of window function width will also affect the frequency resolution. That is, when the width of the window function is wide, the number of communication signals contained on the window function will be 
more, thus increasing the resolution of the frequency. But it will also have an impact on the time resolution, reducing the time resolution ${ }^{[7]}$. Explain the contradiction between temporal and frequency resolution according to the heisenberg uncertainty criterion. The uncertainty criterion can be expressed as:

$$
\Delta t \Delta \omega \geq \frac{1}{2}
$$

In the formula, $\Delta t$ indicates the time period of interception, $\Delta \omega$ indicates frequency hopping bandwidth. As can be seen from the above formula (11), since there is no simultaneous optimal state of temporal and frequency resolution. Therefore, in the practical application, after filtering the different window functions, the appropriate and parameter setting are adopted, and the time resolution and frequency resolution are distributed according to the characteristics of the frequency hopping signal. Through the short-time Fourier transform processing of frequency hopping signal, the uncertain part of non-stationary signal can be analyzed clearly. The time-frequency diagram of signal transformation is shown in the figure below:

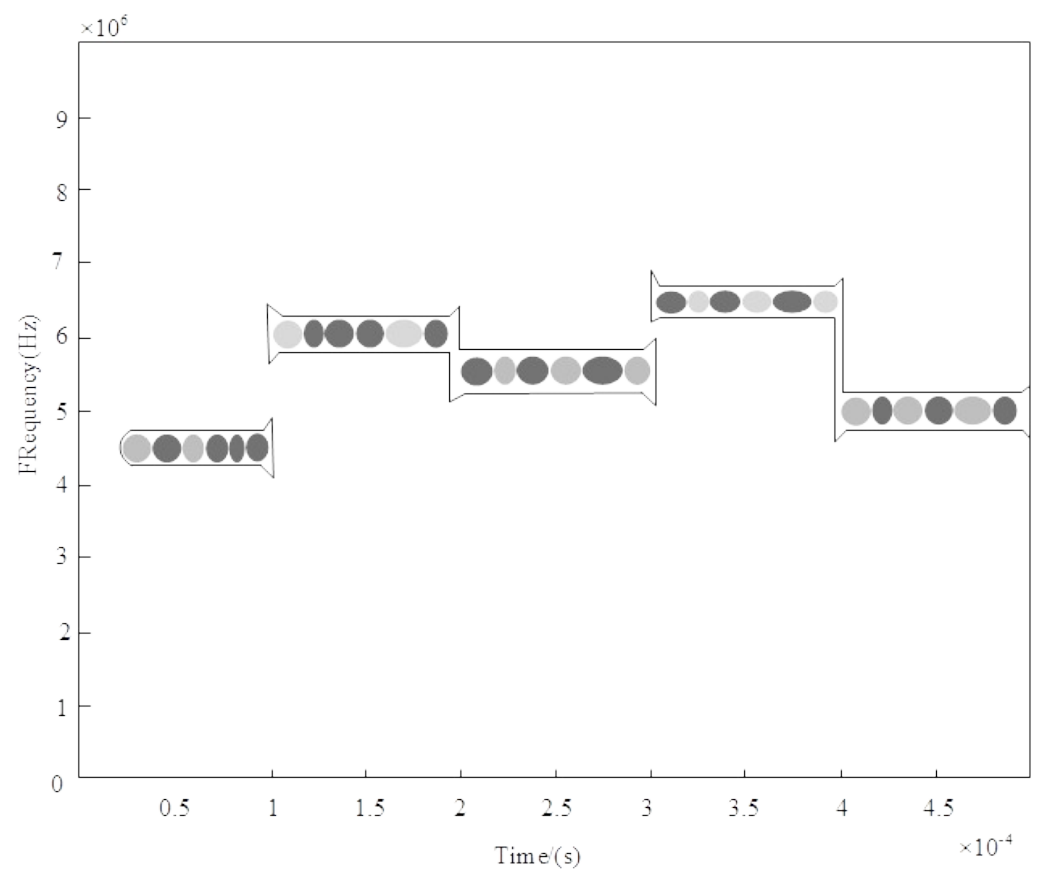

Fig.2 Time-frequency diagram of nonstationary signal short-time Fourier transform

As can be seen from the above figure, the frequency hopping signal is processed by hamming window through short-time Fourier transform. When the width of the 
window function is short, there are fewer points in the spectrum analysis, resulting in lower frequency resolution and higher time resolution. Thus, if there is a frequencyhopping signal, the window function uses a shorter width to locate the frequencyhopping time accurately, however, the frequency resolution is low, and the frequency of the hopping signal will be distributed in a wide band. When the width of the window function is longer, the frequency component of the two time periods before and after the jump will exist simultaneously for a certain period of time, but the frequency distribution of the frequency hopping signal needs to be on a narrower band to make the separation and positioning of the frequency more accurate ${ }^{[8]}$.

\subsection{Blind Separation of Nonstationary Signals}

To realize the underfixed blind separation of nonstationary signal, the underfixed blind separation model is first established. Using array element method, it is assumed that the array element number of nonstationary signal is $M$, A uniform linear array antenna model with a distance of $d$ between two adjacent elements is constructed,In the formula, $\lambda_{\min }$ is expressed as the shortest value of the received signal wavelength, $c$ is the speed of light, $f_{\max }$ is the maximum frequency of the received signal. As shown below: 


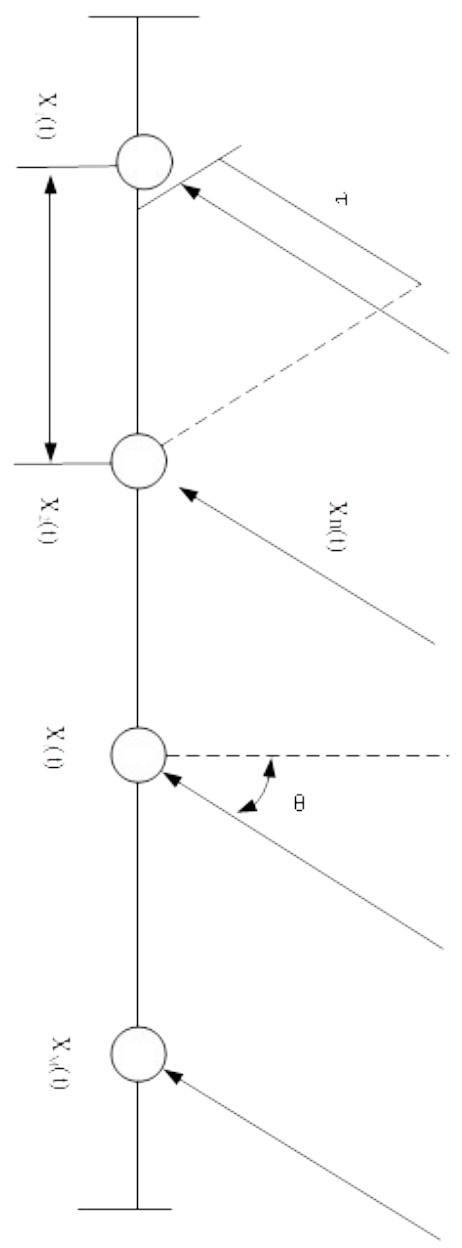

Fig.3 Schematic diagram of uniform linear array antenna

As shown above, the incident angle of the source signal $S_{n}(t)$ is $\theta$, The distance between the two adjacent elements is $d$. Assuming that the transmitter of the nonstationary signal transmits $N$ source signals and that the array number of the receiver is $M$, Select the analytical expression for the nth source signal as follows:

$$
S_{n}(t)=a_{n}(t) e^{j\left[\omega_{n}(t)+\varphi_{n}(t)\right]}
$$

In the formula, $\omega_{n}(t)$ is the source signal frequency function and $\varphi_{n}(t)$ is the source signal phase function. The curves of the two functions are shown below: 


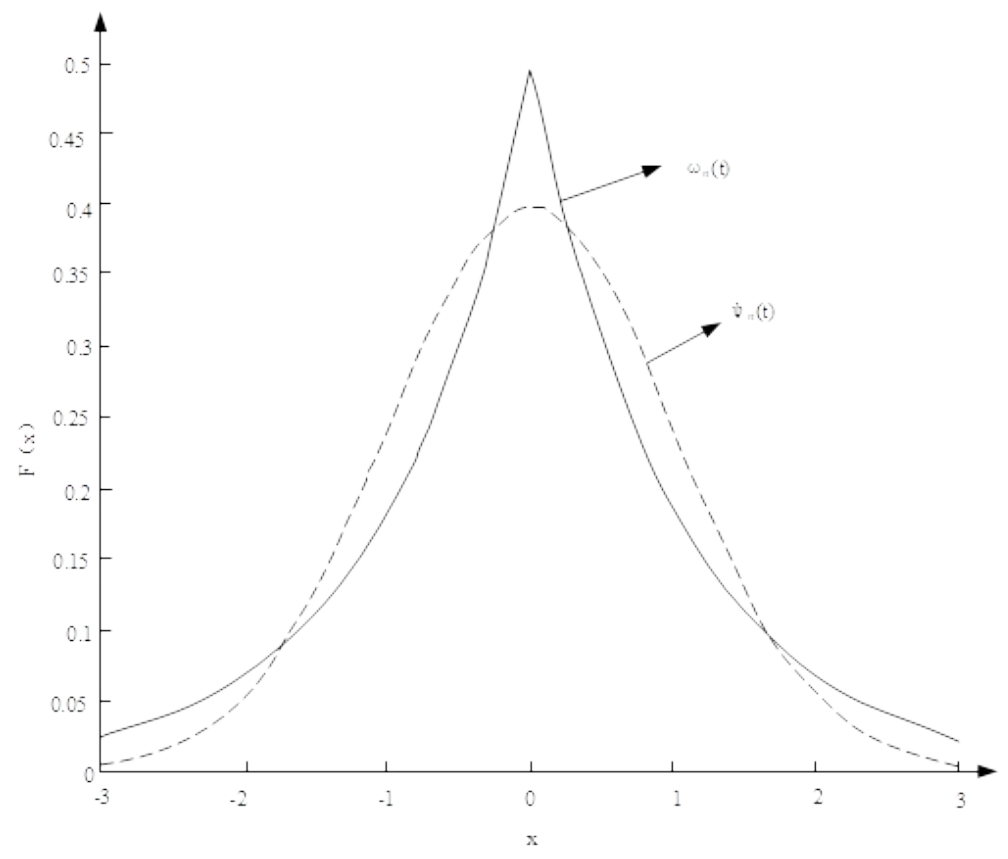

Fig.4 Function change curve of source signal

As shown in the figure above, for fully sparse nonstationary signals, it is assumed that at some point ${ }_{t}$ has a component $S_{m}(t)$ to work, the underdetermined blind separation model can be expressed as:

$$
\left[\begin{array}{c}
x_{1}(t) \\
\vdots \\
x_{M}(t)
\end{array}\right]=\left[\begin{array}{c}
a_{1 n} \\
\vdots \\
a_{M n}
\end{array}\right] S_{m}(t)
$$

The estimation signal is obtained by solving the underdeterministic equation jointly by the upper model ${ }^{[9]}$. There are a large number of non-strictly sparse signals in the actual situation, such as speech signals, which generally adopt sparse transformation, for example, STFT, wavelet transform, Gabo: transform, etc., making it show good sparsity in the transform domain. Two nonstationary signals with a duration of $3 \mathrm{~s}$ and a sampling frequency of $16 \mathrm{kHz}$ are known. The two time domain signals are linearly mixed, iteratively processed, and transformed into frequency domain by STFT. The frequency domain scatter plot is shown in the following figure: 


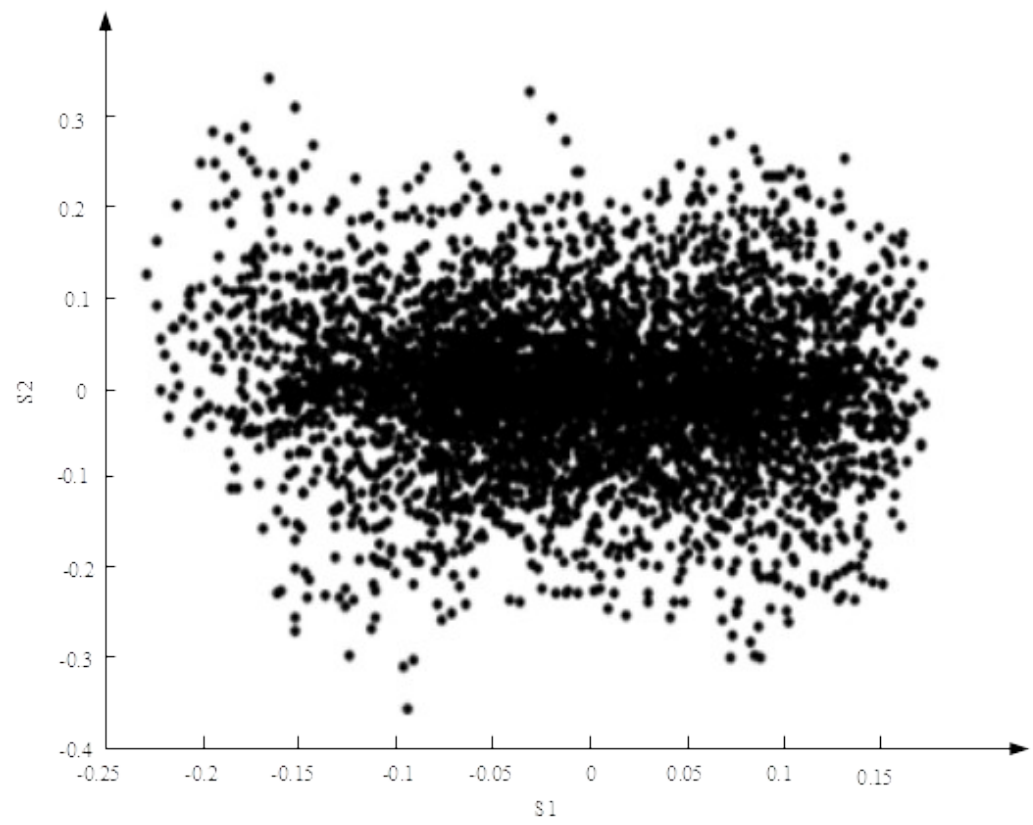

Fig.5 Scatter plot of nonstationary signal in time domain

It can be seen from the graph that the distribution of the time domain scattered point map of speech signal is scattered. The frequency-domain scatter plot is then obtained using iterative STFT processing, as shown in the following figure:

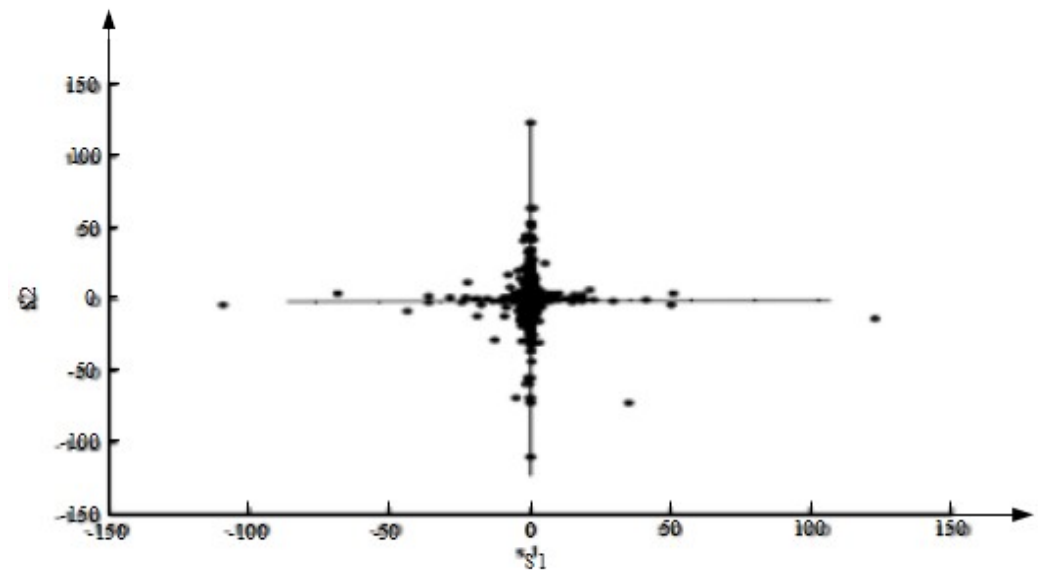

Fig.6 Scatter plot of nonstationary signal in frequency domain

The distribution of frequency domain scatter plot is obviously concentrated on two straight lines, showing obvious clustering characteristics, which indicates that it 
shows good sparsity in frequency domain at this time. The nonstationary signals obtained from the final separation model are shown in the following figure (a) and (b).
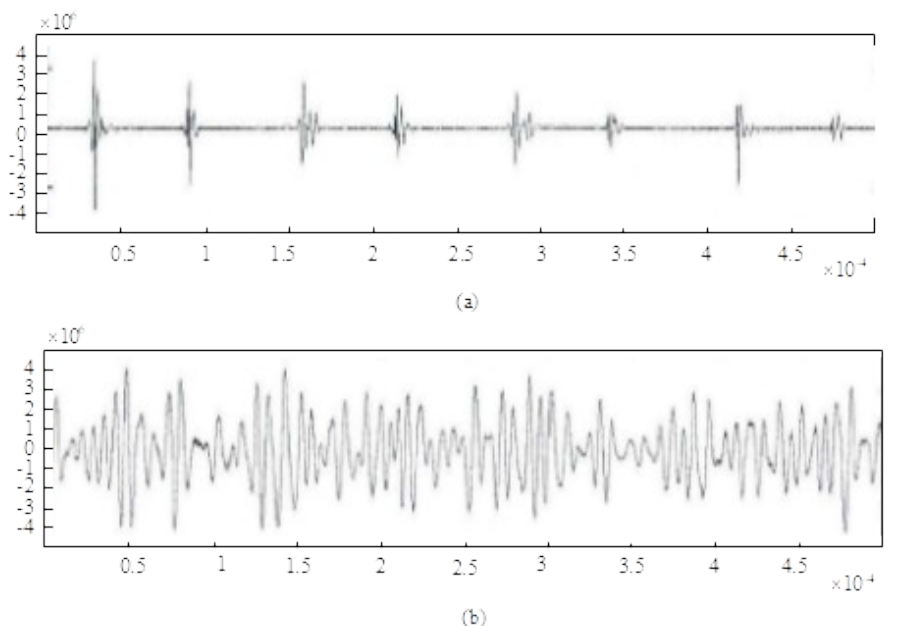

Fig.7 Separation results

As can be seen from the above figure, the spectrum corresponding to S1, S2 and $\mathrm{S} 3$ is in a regular and stable state, and the non-stationary signal underfixed blind separation is realized ${ }^{[10]}$.

\section{Simulation experiment}

\subsection{Experimental Data Set Preparation}

The nonstationary signal is linearly mixed by a hybrid filter matrix of $3 \times 3$ randomly generated by matlab. Following the parameters of the short-time Fourier transform, the mixed signal is processed by means, albinism, pre-weighting and Fourier transform, and the dimension of the mixed signal matrix is $129 \times 3446 \times 2(\mathrm{M} \times$ $\mathrm{N} \times \mathrm{I}$ ). Filter it through the filter, filter processing, where the number of filters $\mathrm{I}=2$, the relevant parameters are: In the case of linear convolution mixing, the initialized mixing matrix $A$ is $129 \times 2 \times 3(M \times I \times J)$, where $J=3$ indicates the number of source signals. The filter length $P$ is 8 , the impulse response matrix of Nonnegative Matrix Factorization (NMF) decomposition is $1 \times 3$, and the 3D nonnegative convolution mixing matrix is initialized. The dimension of randomly initialized $\mathrm{W}$ is $\mathrm{M} \times \mathrm{K}$ and the dimension is $\mathrm{K} \times \mathrm{N}$. In the case of linear instantaneous mixing, the initialized mixing matrix is $2 \times 3(\mathrm{I} \times \mathrm{J})$. the remaining parameter value settings are consistent with the linear convolution case.

The same processing algorithm corresponding to the traditional linear 
instantaneous mixing model is used as the contrast algorithm. The two methods adopt the same data set and performance evaluation criteria. In addition, the improved method in the NMF process, after several test adjustments, considering the feasibility and dimensionality reduction purposes, the dimensionality reduction parameter $\mathrm{k}$ value is taken as 12 .

\subsection{Experimental result}

In order to compare the similarity degree of the signal before and after separation, set the iterative parameter $Z$ to $100-1500$ to take 100 , each run 10 times, take the average value of the correlation coefficient as the reference data. The correlation coefficients between the nonstationary signals of the three separation methods and the separated signals vary with the number of iterations as follows:

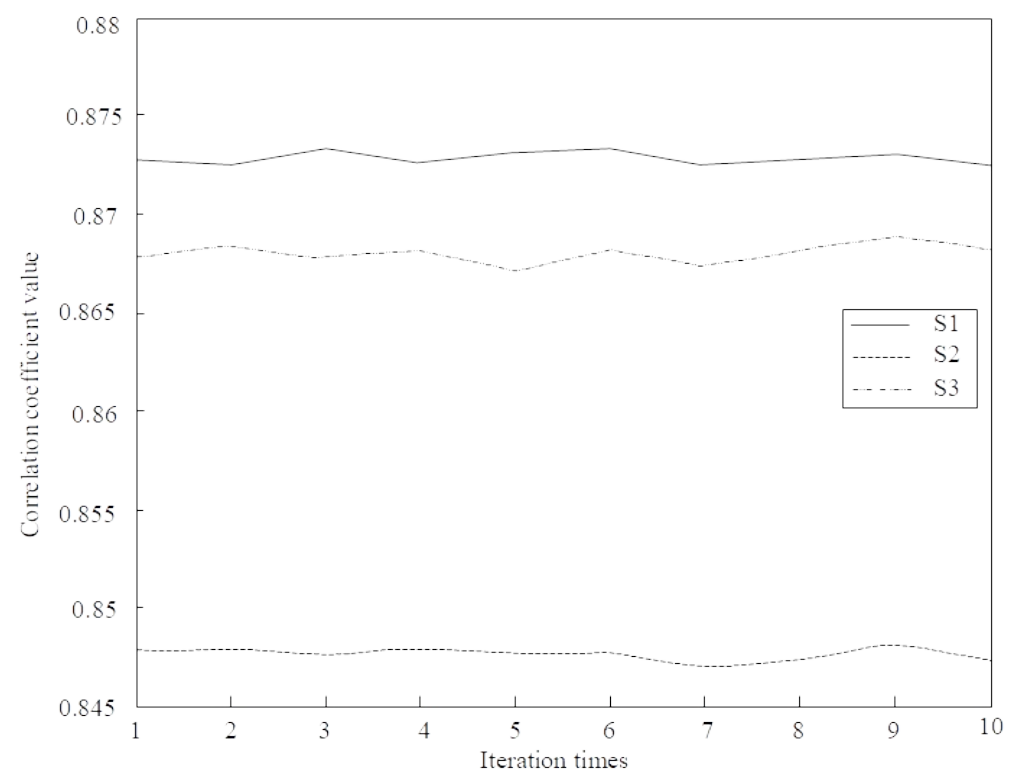

(a) Experimental results of traditional separation method 1 


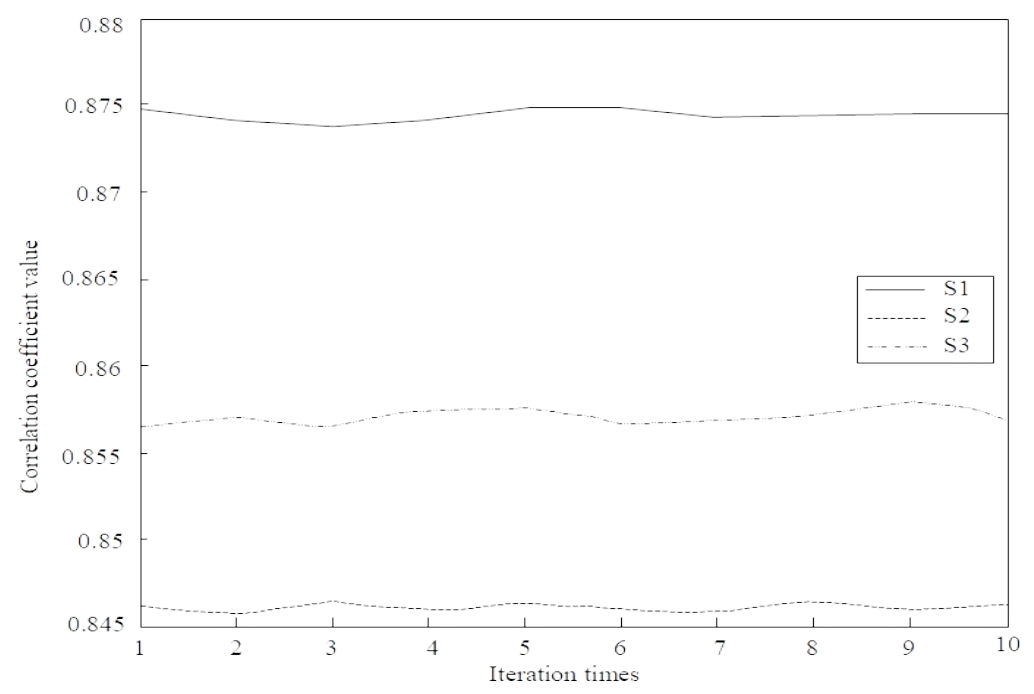

(b) Experimental results of traditional separation method 2

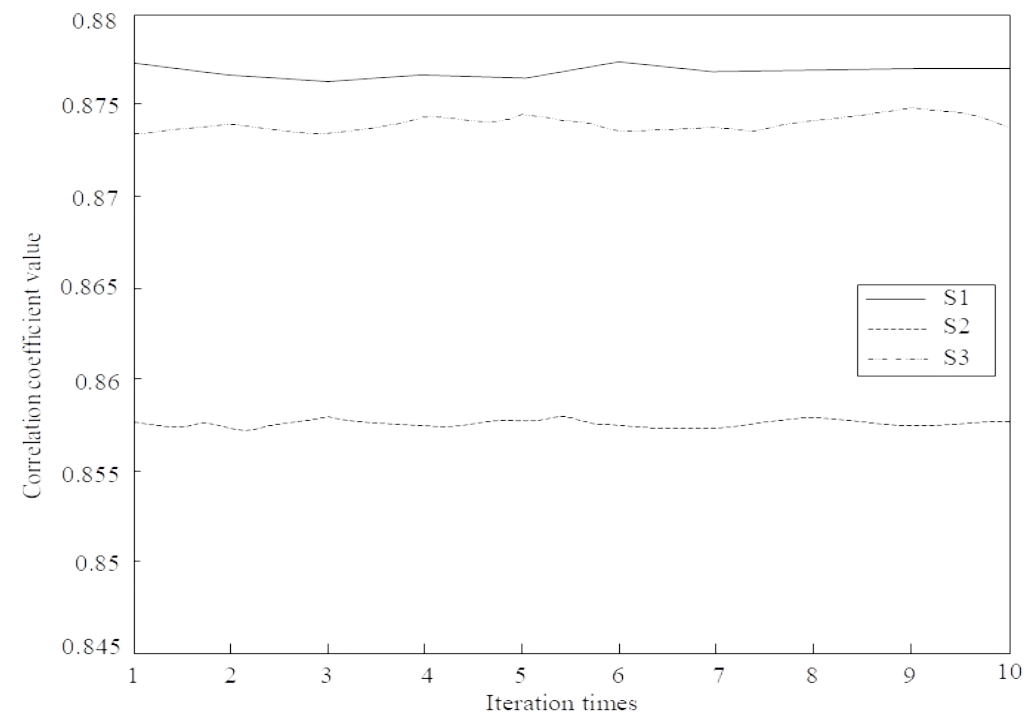

(c) Experimental results of this method

Fig.8 Experimental results of three separation methods

As shown in the above three experimental results, the average value of S1, S2 and $\mathrm{S} 3$ is $0.873,0.867$ and 0.847 respectively. The average iterative coefficient value of S1 of traditional separation method 2 is about 0.875 , and the average iterative coefficient value of $\mathrm{S} 2$ is about 0.858 . The $\mathrm{S} 1$ iteration coefficient is 0.878 , the $\mathrm{S} 2$ iteration coefficient is 0.874 and the $\mathrm{S} 3$ iteration coefficient is 0.858 . The resulting 
iterative coefficients are larger than those obtained by the two separation methods. The results show that the separation method has better performance and improved the smoothness of the correlation coefficient of diagonalization.

\section{Conclusion}

In complex electromagnetic environment, the hybrid signals are overlapped in time domain, frequency domain and even airspace. moreover, because the number of potential source signals is unknown and the number of array elements is limited, the number of sources in the mixed signal is often larger than the number of array elements. At this point, it is very difficult to estimate the parameters and extract information of the mixed signal directly. usually first need to separate the mixed signal under under underfixed conditions. Making full use of the sparsity of the source signal in the time-frequency domain, as well as other statistical characteristics such as independence, cyclic stationary, periodicity and so on, the problem of underfixed blind separation is transformed into a suitable or overfixed problem to complete the separation of the source signal.

\section{Fund project}

Project of Jiangxi Provincial Department of Education, Item number: JXJG-14-812

\section{Reference}

[1] MA Lujia, LAI Wen, ZHAO Xiaobing. Cross-Language Word Embedding Based Query Expansion for Chinese Mongolian Cross-Language Retrieval[J]. Journal of Chinese Information Processing, 2019, 33(6): 27-34.

[2] ZHANG Jingli, ZHOU Wenxuan, HONG Yu,et al. Frame Semantics Based Training Data Expansion for Supervised Event Detecting[J]. Journal of Chinese Information Processing, 2019, 33(5): 82-92+131.

[3] WANG Fei-xin, JIANG Shuai, ZHANG Qing-bo, etal. Calculating method for trajectory and extension of liquid jet in air based on jet-flow theory[J]. Port \& Waterway Engineering, ,2018(07):31-34.

[4] XU Jianmin, LIU Mingyan, WANG Miao. Microblog recommendation method based on extended interest of users[J]. Application Research of Computers ,2019,36(06):1652-16

55.

[5] LI Yue, HAN Wei, CHEN Qingyang, etal. Research on Formation Reconfiguration of UAVs Based on RRT Algorithm[J]. Journal of Northwestern Polytechnical University, 2019,37(03):601-611.

[6] ZHANG Hong-mei, CHU Yuan-feng, XU Guang-yan. UAV Formation Extension 
Consensus Control Based on Joint Errors[J]. Electronics Optics \& Control, 2019,26(08):17-23. [7] HUANG Shan, LI Ming ,CHEN Hao,etal. Visual Clustering Method of Quasi-Circular Mapping Based on Dimension Extension and Rearrangement[J]. Pattern Recognition and Artificial Intelligence, ,2019,32(04):326-335.

[8]LI Wei, ZHENG Hong, CHEN Yuanqiang,et al. Application of the MLS based enriched numerical manifold method in dynamic crack propagation[J]. Chinese Journal of Rock Mechanics and Engineering, 2018,37(07):1574-1585.

[9] JIN Yi-fan, FU Ying-xun, MA Li,etal. Method of Short Text Classification Based on Frequent Item Feature Extension[J]. Computer Science, 2019,46(S1):478-481.

[10] WANG Bo, YAN Xingkui, XU Yuzhe,et al. Retrieving method of multi ocean wave parameters based on GNSS-R technique[J]. Chinese Journal of Radio Science, 2019,34(03):315-321. 